\title{
Cx32 mediates cisplatin resistance in human ovarian cancer cells by affecting drug efflux transporter expression and activating the EGFR-Akt pathway
}

\author{
YU ZHANG $^{1 *}$, LIANG TAO $^{1 *}$, LI-XIA FAN $^{1}$, KUN HUANG $^{1}$, \\ HUI-MIN LUO ${ }^{1}$, HUI GE ${ }^{2}$, XIYAN WANG ${ }^{2}$ and QIN WANG ${ }^{1}$ \\ ${ }^{1}$ Department of Pharmacology, Zhongshan School of Medicine, Sun Yat-Sen University, Guangzhou, \\ Guangdong 510080; ${ }^{2}$ Tumor Research Institute, Xinjiang Medical University Affiliated Tumour Hospital, \\ Urumqi, Xinjiang Uygur Autonomous Region 830000, P.R. China
}

Received May 2, 2018; Accepted November 28, 2018

DOI: $10.3892 / \mathrm{mmr} .2019 .9876$

\begin{abstract}
Our previous study demonstrated that connexin 32 (Cx32) was upregulated and redistributed to the cytoplasm in A2780 human ovarian cancer cells with acquired resistance to cisplatin; this increased $\mathrm{Cx} 32$ feedback promoted cisplatin resistance. To further investigate the mechanism underlying Cx32-mediated cisplatin resistance, alterations in drug transporters, the DNA repair system and the anti-apoptotic signalling pathway were investigated by overexpressing or knocking down Cx32 in parental cells (A2780); cisplatin-resistant human ovarian cancer cells (A2780/CDDP) were also acquired. Upregulation of efflux transporters [multi-drug resistance protein 2 (MRP-2), ATPase copper transporting $\alpha$ (ATP7A) and ATPase copper transporting $\beta$ ] and downregulation of the influx transporter copper uptake protein 1 mediated cisplatin resistance in A2780/CDDP cells. A2780/CDDP cells also exhibited increased expression of the DNA repair enzyme excision repair cross-complementation group 1 (ERCC1) and
\end{abstract}

Correspondence to: Professor Qin Wang, Department of Pharmacology, Zhongshan School of Medicine, Sun Yat-Sen University, 74 Zhongshan 2nd Road, Guangzhou, Guangdong 510080, P.R. China

E-mail: wangqin6@mail.sysu.edu.cn

${ }^{*}$ Contributed equally

Abbreviations: GJ, gap junction; Cx, connexin; MRP-2, multi-drug resistance protein 2; ATP7A, ATPase copper transporting $\alpha$; ATP7B, ATPase copper transporting $\beta$; CTR1, copper uptake protein 1; ERCC1, excision repair cross-complementation group 1; EGFR, epidermal growth factor receptor; p-EGFR, phosphorylated-epidermal growth factor receptor; Akt, protein kinase B; p-Akt, phosphorylated-protein kinase B; Bcl-2, B-cell lymphoma 2; Bak, Bcl-2 antagonist/killer 1; Bax, Bcl-2 associated X

Key words: ovarian cancer, cisplatin resistance, connexin 32, drug efflux transporters, EGFR-Akt signalling pathway activation of the epidermal growth factor receptor (EGFR) signalling pathway. Small interfering RNA-mediated knockdown of Cx32 in A2780/CDDP cells decreased the expression of efflux transporters (MRP-2 and ATP7A). Knockdown of Cx32 in A2780/CDDP cells also decreased the expression of ERCC1, inhibited the activation of the EGFR signalling pathway and enhanced the cytotoxicity of cisplatin. When Cx32 was overexpressed in A2780 cells, an opposite effect on the expression of efflux transporters (MRP-2 and ATP7A) and the activation of the EGFR signalling pathway was observed, which resulted in insensitivity to cisplatin-induced apoptosis. Thus, Cx32 expression may induce cisplatin resistance by modulating drug efflux transporter expression and activating the EGFR-protein kinase B signalling pathway in ovarian cancer cells.

\section{Introduction}

Ovarian cancer poses a serious threat to female health due to its high morbidity and high mortality $(1,2)$. Cisplatin is widely used in chemotherapy as the first-line agent for treating ovarian cancer (3); however, patients are prone to developing drug resistance, which may lead to the failure of cancer treatment $(4,5)$. Our previous study reported that the expression of connexin (Cx)32 was gradually increased during the development of acquired cisplatin resistance in the human ovarian cancer cell line A2780 and that upregulated Cx32 could contribute to cisplatin resistance (6); however, the mechanism underlying Cx32-induced acquired cisplatin resistance requires further investigation.

Gap junctions (GJs) are regarded as tumour suppressors, while Cx, a subunit of GJ, can modulate a series of cellular processes, including proliferation, differentiation and apoptosis, in a GJ-dependent or GJ-independent manner (7-9). Recently, numerous studies have indicated that $\mathrm{Cx}$ contributes to chemoresistance in several types of tumour. Upregulated Cx26 contributed to gefitinib resistance in non-small cell lung cancer cells (10), while increased Cx 26 expression was able to reverse oxaliplatin resistance in hepatocellular carcinoma (11). Upregulation of Cx43 led to the insensitivity 
to temozolomide in glioblastoma cells (12), while increased Cx43 expression enhanced the cytotoxicity of oxaliplatin in colorectal cancer cell lines (13). Our previous study also indicated that $\mathrm{Cx} 32$ promoted cisplatin resistance in human ovarian cancer cells (6); Cx32 inhibited apoptosis in cervical cancer and $(14,15)$.

At present, alterations in drug transporter expression, abnormal activation of the DNA repair system and activation of the anti-apoptotic signalling pathway are believed to be the few main causes of chemoresistance (16-18). Studies on the mechanism of CX-mediated chemoresistance have mainly focused on Cx43. For example, Cx43 expression in SH-SY5Y neuroblastoma cells could inhibit the mitochondrial apoptosis pathway and led to resistance to 1-methyl-4-phenylpyridinium-induced apoptosis (19). Nevertheless, the association between $\mathrm{Cx} 32$ and chemoresistance, and the mechanism by which Cx32 mediates cancer chemoresistance, particularly cisplatin resistance, remains unknown.

The present study aimed to determine the possible mechanisms by which $\mathrm{Cx} 32$ mediates acquired cisplatin resistance in ovarian cancer. Cx32 was knocked down or overexpressed in the ovarian cancer cell line A2780. These cells and a cisplatin-resistant ovarian cancer cell line (A2780/CDDP) were used for the experimentation. The findings of the present study provide insight as to how $\mathrm{Cx} 32$ is involved in cisplatin resistance and proposed $\mathrm{Cx} 32$ as a potential target to effectively reverse cisplatin resistance in human ovarian cancer.

\section{Materials and methods}

Materials. Primary antibodies against Cx32 (cat. no. sc-59948), ATPase copper transporting $\alpha$ (ATP7A; cat. no. sc-376467), and ATPase copper transporting $\beta$ (ATP7B; cat. no. sc-373964) for western blot analysis were purchased from Santa Cruz Biotechnology, Inc. (Dallas, TX, USA). Primary antibodies against multi-drug resistance protein 2 (MRP-2; cat.no. ab3373) and copper uptake protein 1 (CTR1; cat. no. ab129067) for western blotting were purchased from Abcam (Cambridge, UK). Primary antibodies against excision repair cross-complementation group 1 (ERCC1; cat. no. 5437), epidermal growth factor receptor (EGFR; cat. no. 4267), phosphorylated-EGFR (p-EGFR; cat. no. 2236), protein kinase B (Akt; cat. no. 4685), p-Akt (cat. no. 4060), cleaved caspase-3 (cat. no. 9664), B-cell lymphoma 2 (Bcl-2; cat. no. 4223), Bcl-2 associated X (Bax; cat. no. 5023), Bcl-2 antagonist/killer 1 (Bak; cat. no. 12105) and the cell lysis buffer (cat. no. 9803) were purchased from Cell Signaling Technology, Inc. (Danvers, MA, USA). Primary antibodies against tubulin (cat. no. T4026) for western blot analysis and cisplatin were purchased from Sigma-Aldrich (Merck KGaA, Darmstadt, Germany). Secondary antibodies, including goat anti-mouse IgG (cat. no. 115-005-003) and goat anti-rabbit IgG (cat. no. 111-035-003) for western blot analysis were purchased from Jackson ImmunoResearch Laboratories, Inc. (West Grove, PA, USA). Cell culture-associated reagents and Lipofectamine ${ }^{\mathrm{TM}} 3000$ were purchased from Invitrogen (Thermo Fisher Scientific, Inc., Waltham, MA, USA). The fluorescein isothiocyanate (FITC) Annexin V Apoptosis Detection kit was purchased from BD Biosciences (San Jose, CA, USA). Cx32 small interfering (si)RNA was constructed by Guangzhou RiboBio Co., Ltd. (Guangzhou, China). The empty plasmid (cat. no. EX-EGFP-M02) and the plasmid overexpressing Cx32 (cat. no. EX-A0514-M02-5) were constructed by Genecopoeia, Inc. (Rockville, MD, USA). All other reagents were purchased from Sigma-Aldrich (Merck KGaA) unless otherwise stated.

Cell culture. The A2780 cell line was originally purchased from the American Type Culture and Collection (Manassas, VA, USA). The cisplatin-resistant cell line A2780/CDDP was generated in our previous study (6). A2780 and A2780/CDDP cells were routinely cultured in Dulbecco's Modified Eagle's medium (DMEM; Thermo Fisher Scientific, Inc.) containing $10 \%$ foetal bovine serum (Thermo Fisher Scientific, Inc.) and $1 \%$ penicillin-streptomycin (Thermo Fisher Scientific, Inc.) at $37^{\circ} \mathrm{C}$ in a $5 \% \mathrm{CO}_{2}$ atmosphere.

To induce apoptosis, A2780 and A2780/CDDP cells were exposed to 5 and $15 \mu \mathrm{g} / \mathrm{ml}$ cisplatin for $24 \mathrm{~h}$, respectively.

Assessment of cell viability. A2780 or A2780/CDDP cells were plated at $5 \times 10^{3}$ cells/well in 96 -well plates. Cells were treated with a concentration series $(0,0.3,1,3,10,30$ and $100 \mu \mathrm{g} / \mathrm{ml}$ ) of cisplatin at $37^{\circ} \mathrm{C}$ for $48 \mathrm{~h}$, and the untreated cells served as the negative control. Cell viability was assessed using a Cell Counting Kit-8 assay (CCK-8; Dojindo Molecular Technologies, Inc., Kumamoto, Japan). After a 1-h incubation with CCK-8 reagent, the plate was placed in a microplate reader (BioTek Instruments, Inc., Winooski, VT, USA) and the absorbance was determined at a wavelength of $450 \mathrm{~nm}$.

The resistance index (RI) was used to test the degree of cisplatin resistance, and it was determined by the ratio of the $\mathrm{IC}_{50}$ of drug-resistant cells to the $\mathrm{IC}_{50}$ of sensitive cells.

Western blot analysis. For western blot analysis, the cells were washed with ice-cold PBS, lysed by cell lysis buffer (Cell Signaling Technology, Inc.) and centrifuged at $4^{\circ} \mathrm{C}$ and $12,000 \mathrm{x} \mathrm{g}$ for $30 \mathrm{~min}$. Total protein concentration was quantified using a BCA assay kit (Bio-Rad Laboratories, Inc., Hercules, CA, USA). Subsequently, a total of $20 \mu \mathrm{g}$ protein was loaded in each lane. Proteins were separated by $10 \%$ SDS-PAGE or, for the detection of cleaved caspase-3, by $12 \%$ SDS-PAGE. Proteins were transferred onto a polyvinylidene difluoride membrane. The membranes were blocked with TBS containing 5\% dry milk and $0.1 \%$ Tween-20 at $25^{\circ} \mathrm{C}$ for $2 \mathrm{~h}$. Following blocking, specific primary antibodies were applied to the membranes at $4^{\circ} \mathrm{C}$ for $16 \mathrm{~h}$. The monoclonal antibodies were diluted with TBS containing $0.1 \%$ Tween-20 as follows: Anti-Cx32 (1:2,000), anti-MRP-2 (1:500), anti-ATP7A (1:1,000), anti-ATP7B (1:1,000), anti-CTR1 (1:1,000), anti-ERCC1 (1:1,000), anti-EGFR $(1: 1,000)$, anti-p-EGFR (1:1,000), anti-Akt (1:1,000), anti-p-Akt (1:1,000), anti-cleaved caspase-3 (1:1,000), anti-Bcl-2 (1:1,000), anti-Bak $(1: 1,000)$, anti-Bax $(1: 1,000)$ and anti-tubulin $(1: 10,000)$. The membranes were incubated with the relevant horseradish peroxidase-conjugated secondary antibodies (diluted in TBS containing $0.1 \%$ Tween- $20 ; 1: 2,000)$ at room temperature for $1 \mathrm{~h}$. The membranes were subsequently incubated with Immobilon $^{\text {TM }}$ Western Chemiluminescent HRP Substrate (EMD Millipore, Billerica, MA, USA) and immunoreactive bands were detected by ImageQuant LAS 4000 (GE Healthcare Life Sciences, Little Chalfont, UK). Band density 
was analysed by Image J software (version 1.46r; National Institutes of Health, Bethesda, MD, USA). Each experiment was repeated at least three times.

Plasmid transfection and siRNA interference experiments. For Cx32 overexpression (Plasmid-Cx32), A2780 cells were plated at $3 \times 10^{5}$ cells/well in 6 -well plates. A total of $4 \mu \mathrm{g}$ Plasmid-Cx32 was mixed with Lipofectamine ${ }^{\circledR} 3000$ reagent. The mix was added to the cells in DMEM. After $48 \mathrm{~h}$, expression of Cx32 was detected by western blot analysis. A2780 cells transfected with $4 \mu \mathrm{g}$ empty vector served as the control group.

Cx32 siRNA interference (knockdown Cx32) was performed in a similar manner as plasmid transfection. A2780/CDDP cells were plated at $1.5 \times 10^{5}$ cells/well in 6-well plates. Lipofectamine 3000 was mixed with the siRNAs at a concentration of $50 \mathrm{nM}$ and added to the cell medium. After $48 \mathrm{~h}$, the levels of Cx32 expression were assessed by western blot analysis. The sequences for the negative control (NC) siRNA and siRNAs targeting Cx32 (siRNA-Cx32) were as follows: siRNA-NC: 5'-UUCUCCGAACGUGUC ACGUTT-3'; siRNA-Cx32_1: 5'-CCGGCATTCTACTGC CATT-3'; siRNA-Cx32_2: 5'-GGCTCACCAGCAACA CATA-3' and siRNA-Cx32_3: 5'-GCAACAGCGTTTGCT ATGA-3'. A2780/CDDP cells transfected with siRNA_NC at a concentration of $50 \mathrm{nM}$ served as the control group. The siRNA-Cx32_3 was used for subsequent knockdown Cx32 experiments.

Flow cytometry for apoptosis analysis. A2780 and A2780/CDDP cells were seeded in six-well plates and cultured with $5 \mu \mathrm{g} / \mathrm{ml}$ cisplatin at $37^{\circ} \mathrm{C}$ for $36 \mathrm{~h}$. Untreated A2780 and A2780/CDDP cells served as the control for treated A2780 and treated A2780/CDDP, respectively. Cells were washed with PBS and trypsinised with $0.05 \%$ trypsin at $37^{\circ} \mathrm{C}$ for $2 \mathrm{~min}$. Subsequently, harvested cells were centrifuged at 2,000 x $\mathrm{g}$ for $5 \mathrm{~min}$ and resuspended in binding buffer (provided in the FITC Annexin V Apoptosis Detection kit) mixed with FITC-Annexin $\mathrm{V}$ and propidium iodide (PI) according to the manufacturer's protocols. The data were detected and analyzed using the flow cytometer BriCyte E6 and the software MRFlow (Shenzhen Mindray Bio-Medical Electronics Co., Ltd., Shenzhen, China) within $1 \mathrm{~h}$ after cells were stained.

Statistical analysis. All experiments were repeated at least three times. Parametric data were analysed using an unpaired Student's t-test or two-way analysis of variance using GraphPad Prism 6.0 software (GraphPad Software, Inc., La Jolla, CA, USA). A Bonferroni post hoc test was applied for multiple comparisons. The results are expressed as the mean \pm standard error of the mean. $\mathrm{P}<0.05$ was considered to indicate a statistically significant difference.

\section{Results}

Drug resistance analysis in an acquired cisplatin-resistant human ovarian cancer cell line (A2780/CDDP). The A2780/CDDP cells were established by the stepwise selection of A2780 cells cultured in growth media with increasing cisplatin concentrations (6). Several experiments were performed to determine the drug resistance of A2780/CDDP cells.

The CCK-8 assay revealed that the RI of A2780/CDDP cells was 5.105 (Fig. 1A). A2780 and A2780/CDDP cells were treated with $5 \mu \mathrm{g} / \mathrm{ml}$ cisplatin to induce apoptosis. Western blot analysis revealed that the expression levels of cleaved caspase-3 in A2780/CDDP cells were lower than that of A2780 cells (Fig. 1B and C). In addition, flow cytometry analysis demonstrated the significantly enhanced resistance of A2780/CDDP cells to cisplatin-induced apoptosis compared with in the control and A2780 cells (Fig. 1D and E).

Cx32 participates in cisplatin resistance by affecting the expression of drug efflux transporters. To demonstrate the effects of $\mathrm{Cx} 32$ on cisplatin resistance, the association between $\mathrm{Cx} 32$ and three factors associated with drug resistance was investigated. We compared the expression levels of several drug resistance-associated proteins between A2780 cells and A2780/CDDP cells. After altering the expression of $\mathrm{Cx} 32$, the expression levels of numerous drug resistance-associated proteins were detected. In siRNA experiments, interference fragment S3 (siRNA-Cx32_3) significantly decreased the expression of Cx32 in A2780/CDDP cells compared with the negative control (Fig. 2A and B). Therefore, in subsequent siRNA interference experiments, siRNA-Cx32_3 (siRNA-Cx32) was selected to target $\mathrm{Cx} 32$ expression.

A statistically significant increased expression of efflux transporters was observed, including MRP-2, ATP7A and ATP7B, in A2780/CDDP cells, while the expression of CTR1 (an influx transporter) was significantly lower in A2780/CDDP cells than in A2780 cells (Fig. 2C and D).

Following overexpression of Cx32 in A2780 cells, the expression levels of MRP-2 and ATP7A were significantly increased, while the levels of ATP7B and CTR1 exhibited no significant difference compared with the control (Fig. 2E and F). However, following Cx32 knockdown in A2780/CDDP cells, the expression levels of MRP-2 and ATP7A were significantly decreased, while the levels of ATP7B and CTR1 exhibited no significant difference compared with the control (Fig. 2G and $\mathrm{H}$ ).

Activation of the DNA repair system does not involve the mechanism of Cx32-mediated cisplatin resistance. The results revealed a statistically significant increase in ERCC1 expression in A2780/CDDP cells compared with in A2780 cells (Fig. 3A and B). Overexpression of Cx32 in A2780 cells and knockdown of Cx32 in A2780/CDDP cells significantly decreased the protein expression levels of ERCC1 compared with the control (Fig. 3C-F). The present results suggested that the activation of the DNA repair system may not be the principal mechanism of Cx32-mediated chemoresistance to cisplatin in ovarian cancer.

Cx32 can protect against cell apoptosis induced by cisplatin. Following Cx32 overexpression using plasmid-Cx32 in A2780 cells, these cells were exposed to cisplatin for 48 h. The expression levels of cleaved caspase-3 were detected 
A

$\mathrm{RI}=\frac{\mathrm{IC} \mathrm{C}_{50}(\mathrm{~A} 2780 / \mathrm{CDDP})}{\mathrm{IC}_{50}(\mathrm{~A} 2780)}$

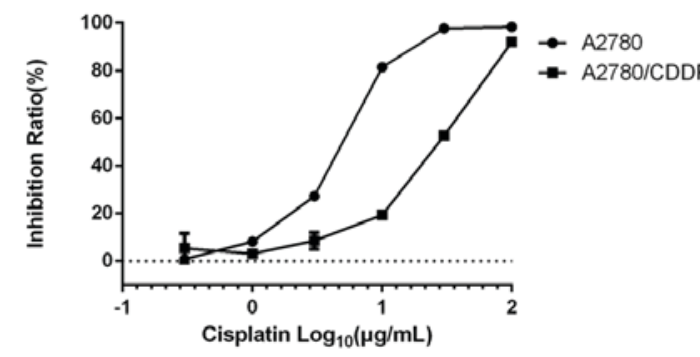

B

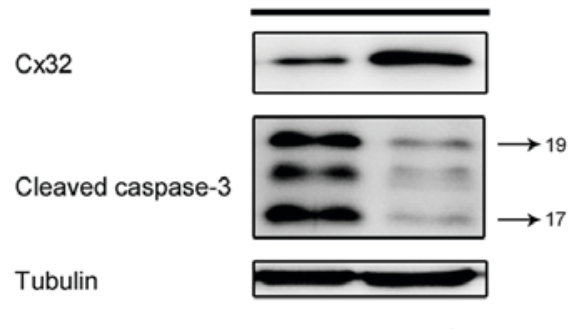

A2780 A2780/CDDP
C

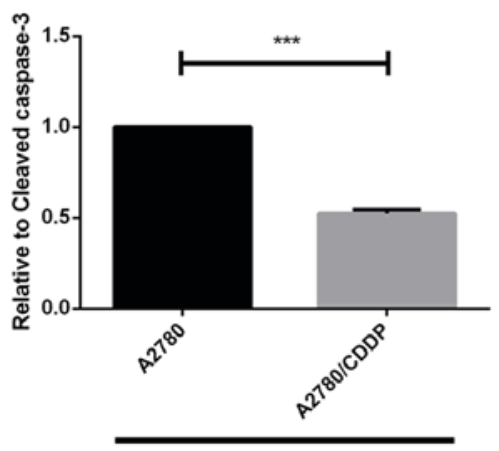

Cisplatin

D
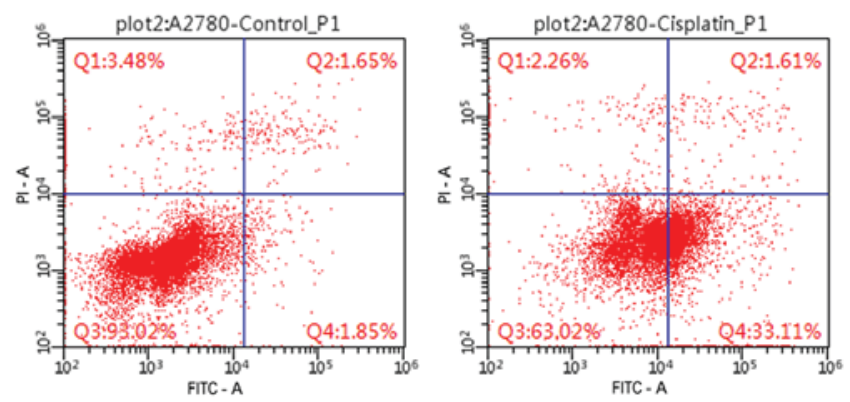

$\mathrm{E}$
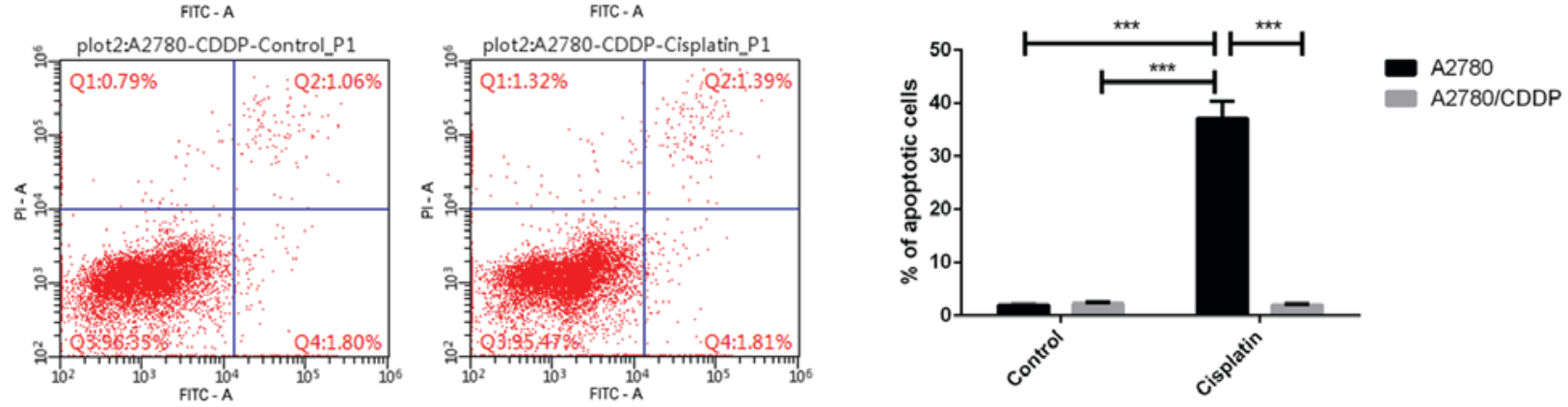

Figure 1. Drug resistance analysis in an acquired CDDP-resistant human ovarian cancer cells, A2780/CDDP. (A) A2780 cells and A2780/CDDP cells were treated with a series of concentrations of cisplatin for $48 \mathrm{~h}$, and the inhibition ratio of cell growth was determined by a Cell Counting Kit- 8 assay ( $\mathrm{n}=3$ ). (B) Following treatment with $5 \mu \mathrm{g} / \mathrm{ml}$ cisplatin for $24 \mathrm{~h}$, the expression levels of cleaved caspase- 3 was detected by western blotting $(\mathrm{n}=3)$. (C) Densitometric analysis. (D) A2780 and A2780/CDDP cells were exposed to $5 \mu \mathrm{g} / \mathrm{ml}$ cisplatin for $36 \mathrm{~h}$, and the (E) percentage of apoptotic cells was detected by flow cytometry $(\mathrm{n}=3)$; quantitative results are presented. ${ }^{* * *} \mathrm{P}<0.001$. Error bar, standard error. CDDP, cisplatin; Cx32, connexin 32 ; $\mathrm{IC}_{50}$, half-maximal inhibitory concentration; FITC, fluorescein isothiocyanate; RI, resistance index.

by western blot analysis and the cell inhibition ratio was determined by a CCK- 8 assay. The results revealed that the sensitivity to cisplatin and the expression levels of cleaved caspase-3 significantly decreased upon Cx32 overexpression compared with the control (Fig. 4A-C). Furthermore, the protein expression levels of three members of the Bcl-2 family (Bcl-2, Bak and Bax) were analysed. Following transfection of plasmid-Cx32, the expression of anti-apoptotic Bcl-2 was significantly increased, while that of the pro-apoptotic members Bak and Bax was decreased compared with the control (Fig. 4D and E). On the contrary, Cx32 knockdown was performed in A2780/CDDP cells via siRNA-Cx32; the cells were then treated with cisplatin for $48 \mathrm{~h}$. Cx32 knockdown significantly increased cisplatin cytotoxicity in response to $0.3,1,3$ and $10 \mu \mathrm{g} / \mathrm{ml}$ cisplatin; the expression of cleaved caspase- 3 was significantly upregulated compared with the control (Fig. 4F-H). When the expression of $\mathrm{Cx} 32$ was reduced, alterations in the expression of the three $\mathrm{Bcl}-2$ protein family members revealed opposing results to that of Cx32 overexpression (Fig. 4I and J).

Cx32 promotes cisplatin resistance by activating the EGFR-Akt signalling pathway. Activation of the EGFR signalling pathway may inhibit cell apoptosis (14). In the present study, a statistically significant increase in EGFR, p-EGFR, and p-Akt expression in A2780/CDDP cells compared with in A2780 cells was observed (Fig. 5A and B). To further investigate the anti-apoptotic effects of $\mathrm{Cx} 32$ in cisplatin resistance, it was proposed that Cx32 may activate the EGFR signalling pathways. Thus, we overexpressed Cx32 in A2780 cells not treated with cisplatin; the expression of EGFR and downstream Akt was analysed by western blot analysis. The results revealed that the expression levels of $\mathrm{p}-\mathrm{EGFR}$ and p-Akt were upregulated, and the ratios of p-EGFR/EGFR 
A

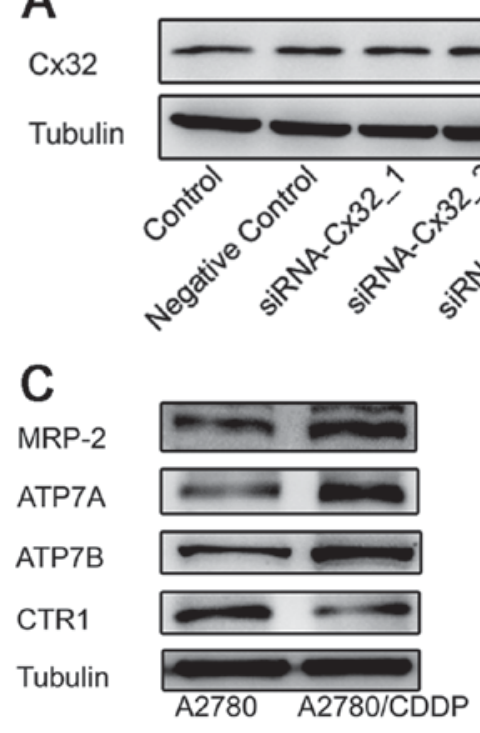

E

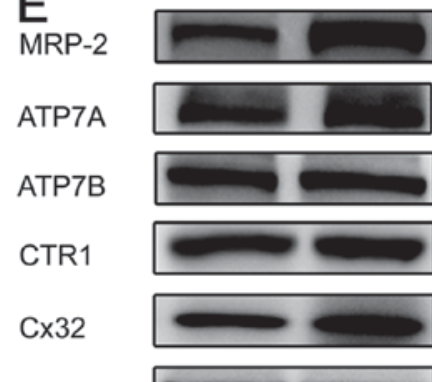

Tubulin
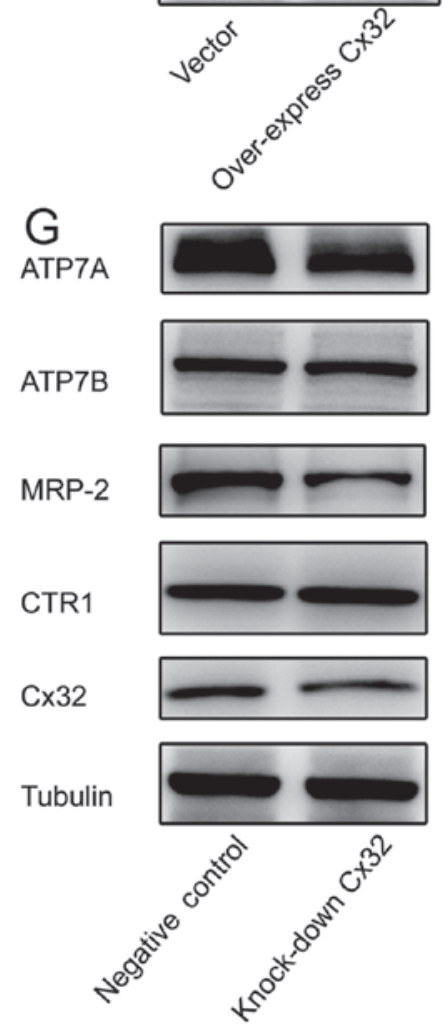
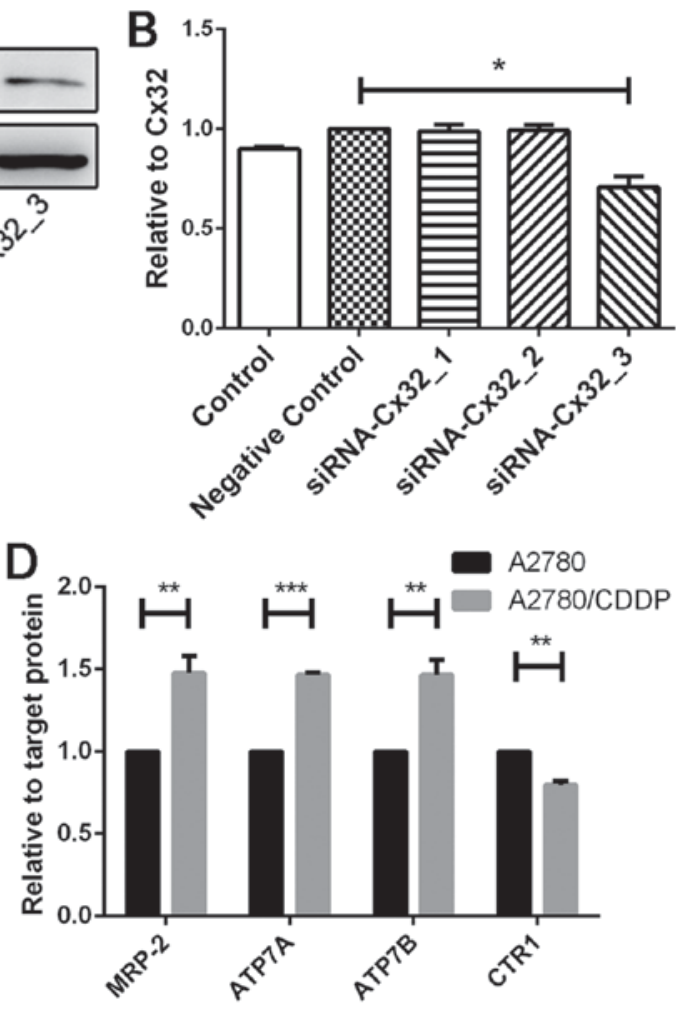

$\mathbf{F}$

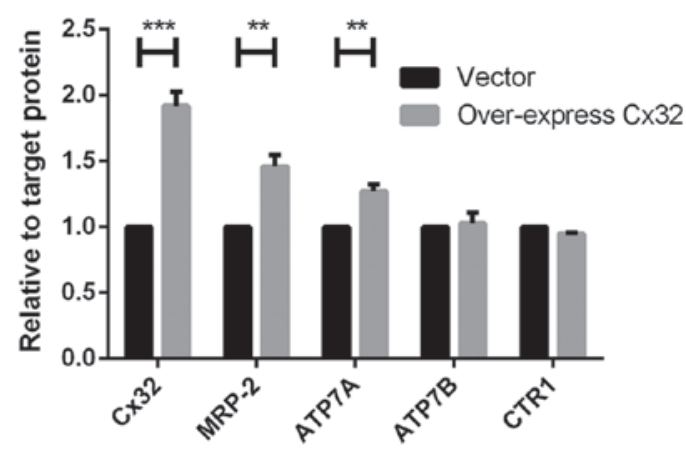

$\mathrm{H}$

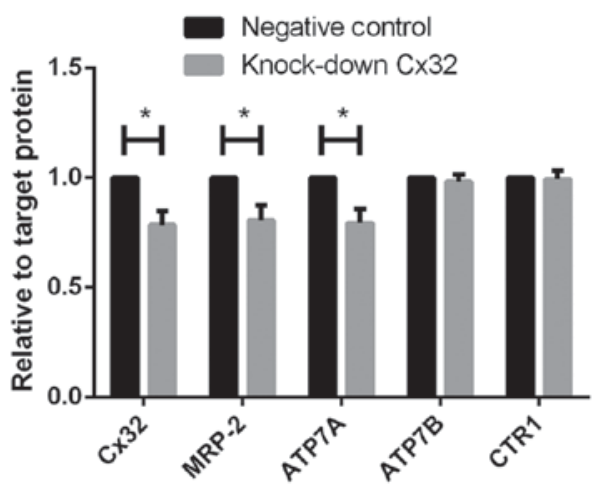

Figure 2. Cx32 participates in CDDP resistance by affecting the expression of drug efflux transporters. (A and B) A2780/CDDP cells were transfected with siRNA targeting $\mathrm{Cx} 32$, and the expression levels of $\mathrm{Cx} 32$ were detected by western blotting $(\mathrm{n}=3)$. (C and $\mathrm{D})$ Numerous $\mathrm{CDDP}$ transport-associated proteins were detected by western blotting ( $\mathrm{n}=3$ ). A2780/CDDP cells exhibited increased expression of MRP-2, ATP7A and ATP7B, but decreased expression of CTR1. (E and F) Cx32 was overexpressed in A2780 cells; cisplatin transporters were detected by western blotting (n=3). ( $\mathrm{G}$ and $\mathrm{H})$ Cx32 knockdown by siRNA-Cx32 in A2780/CDDP cells; cisplatin transport-associated proteins were detected by western blotting $(\mathrm{n}=3) .{ }^{*} \mathrm{P}<0.05,{ }^{* *} \mathrm{P}<0.01,{ }^{* * *} \mathrm{P}<0.001$ Error bar, standard error. ATP7A, ATPase copper transporting $\alpha$; ATP7B, ATPase copper transporting $\beta$; CDDP, cisplatin; Cx32, connexin 32; CTR1, copper uptake protein 1; MRP-2, multi-drug resistance protein 2; siRNA, small interfering RNA. 

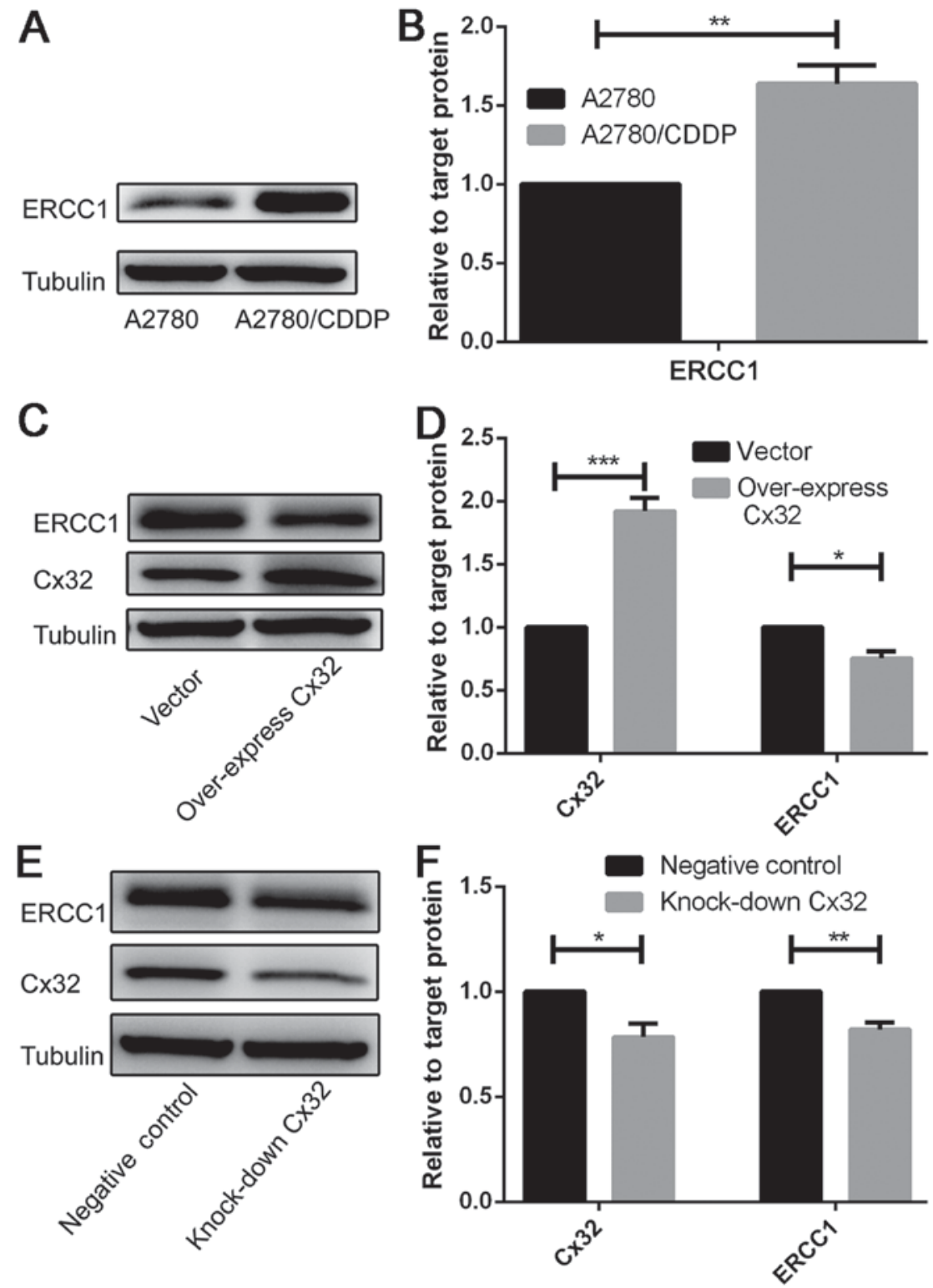

Figure 3. Activation of the DNA repair system does not involve the mechanism of Cx32-mediated CDDP resistance. (A and B) ERCC1 was detected by western blotting ( $\mathrm{n}=3$ ). A2780/CDDP cells demonstrated increased expression of ERCC1. (C and D) Cx32 was overexpressed using plasmid-Cx32 in A2780 cells; ERCC1 was detected by western blotting $(\mathrm{n}=3)$. (E and F) Cx32 knockdown by small interfering RNA-Cx32 in A2780/CDDP cells; ERCC1 was detected by western blotting $(\mathrm{n}=3)$. ${ }^{*} \mathrm{P}<0.05,{ }^{* *} \mathrm{P}<0.01,{ }^{* * * *} \mathrm{P}<0.001$. Error bar, standard error. CDDP, cisplatin; Cx32, connexin 32; ERCC1, excision repair cross-complementation group 1 .

and $\mathrm{p}-\mathrm{Akt} / \mathrm{Akt}$ were significantly increased compared with the control (Fig. 5C-E). This suggested that the EFGR-Akt signalling pathway was activated. Additionally, Cx32 was knocked down in A2780/CDDP cells and the EFGR-Akt pathway was investigated. The results demonstrated that the expression levels of EGFR and p-Akt were significantly downregulated, and the ratio of p-Akt/Akt was significantly decreased compared with the control (Fig. 5F-H). This indicated that activation of the EFGR-Akt signalling pathway was suppressed.

\section{Discussion}

In clinical practice, cisplatin is a first-line chemotherapeutic drug for the treatment of ovarian cancer, which threatens women's health due to its high rate of morbidity and mortality $(2,3)$; however, during the course of treatment, drug resistance may be acquired, which leads to treatment failure. Our prior research has determined that $\mathrm{Cx} 32$ could serve an anti-apoptotic role independent of GJ in human cervical cancer cells, which suggests that $\mathrm{Cx} 32$ may contribute to chemoresistance (14). However, our previous study on non-drug-resistant cells did not demonstrate that $\mathrm{Cx} 32$ was involved in chemoresistance (14). Additionally, a two-fold increase in the expression of $\mathrm{Cx} 32$ and the functional inhibition of GJ was observed, which occurred when A2780 cells acquired cisplatin resistance (RI=7.1) (6). Thus, studies on cisplatin-resistant cells (A2780/CDDP) may further demonstrate how $\mathrm{Cx} 32$ is involved in cisplatin resistance.

At present, numerous factors have been proposed to affect cisplatin resistance, including drug transporters, the DNA repair system and the anti-apoptotic signalling pathway $(18,20)$. 
A

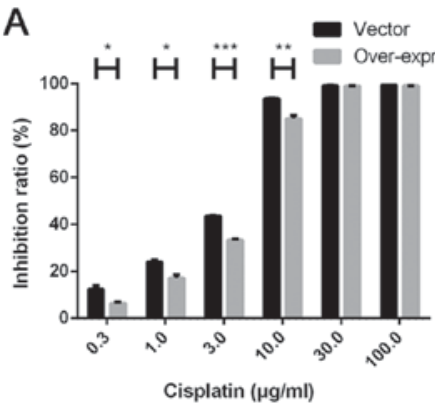

$\mathbf{D}_{\mathrm{c} \times 32}$

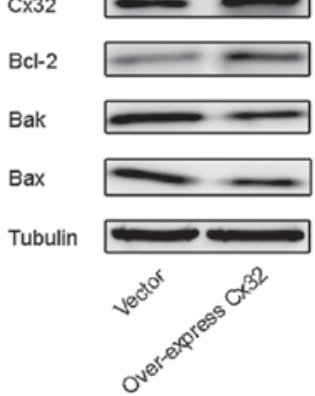

F

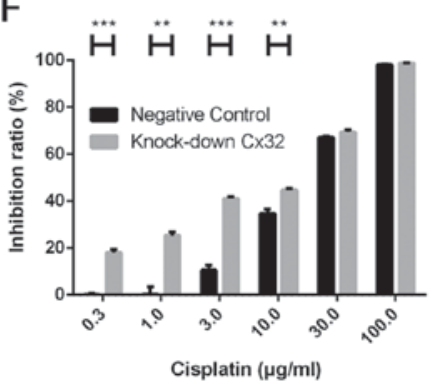

B

C $\times 32$

Cleaved caspase-3

Tubulin

G

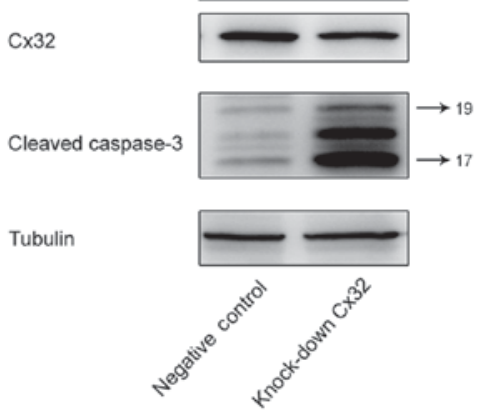

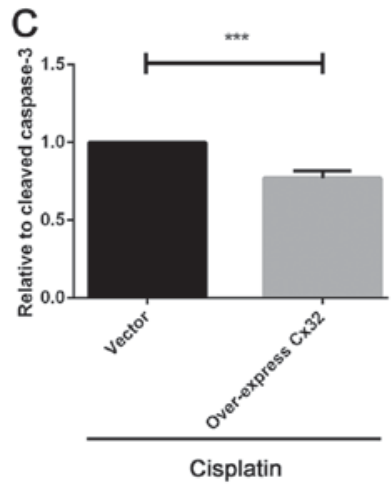

E
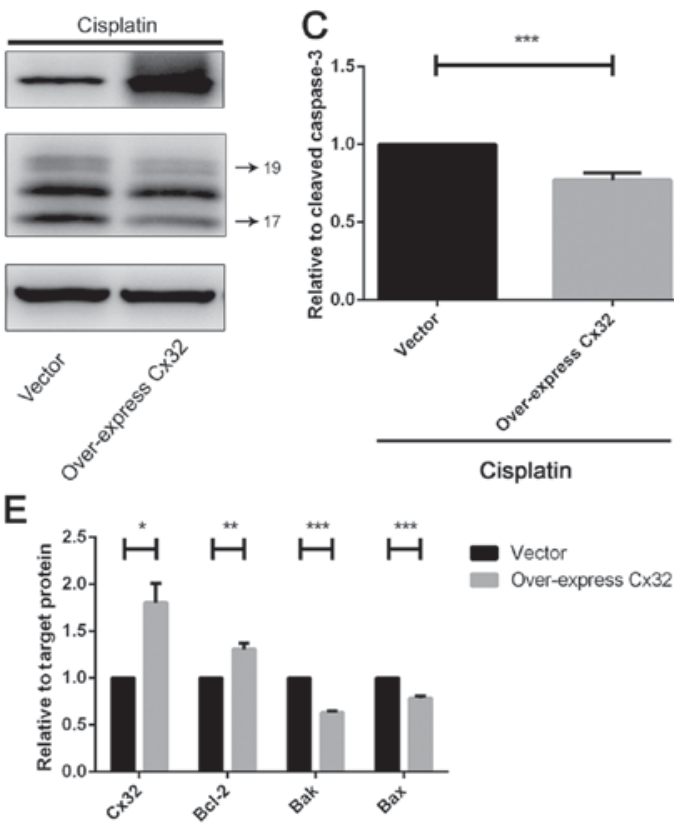

H

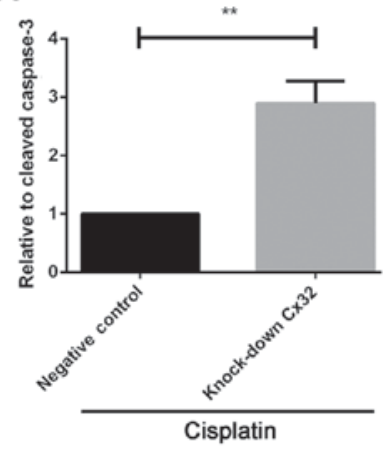

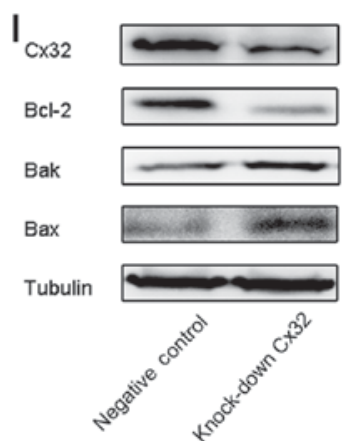

J

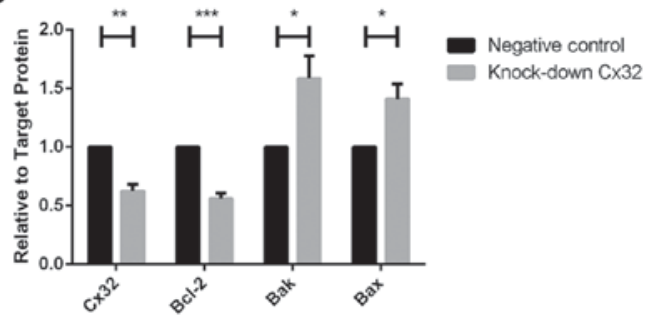

Figure 4. Cx32 can protect against cell apoptosis induced by CDDP. (A) Following overexpressing Cx32, A2780 cells were treated with a concentration series of CDDP. After 48 h, cell viability was determined by the CCK-8 assay ( $\mathrm{n}=3$ ). (B and C) Cx32 was overexpressed via plasmid-Cx32 in A2780 cells. The cells were treated with CDDP $(5 \mu \mathrm{g} / \mathrm{ml})$ for $24 \mathrm{~h}$. Cleaved caspase-3 expression was detected by western blotting ( $\mathrm{n}=3$ ). (D and E) Following Cx32 overexpression, Bcl-2 family members (Bcl-2, Bak and Bax) were analysed by western blotting (n=3). (F) Following Cx32 knockdown, A2780/CDDP cells were treated with a concentration series of CDDP. After 48 h, cell viability was determined by a CCK-8 assay. (G and H) Cx32 was downregulated by siRNA-Cx32 in A2780/CDDP cells. The cells were treated with CDDP $(15 \mu \mathrm{g} / \mathrm{ml})$ for $24 \mathrm{~h}$. Cleaved caspase-3 was detected by western blotting (n=3). (I and J) Cx32 was downregulated by siRNA-Cx32; the aforementioned Bcl-2 family members were detected by western blotting $(\mathrm{n}=3)$. ${ }^{*} \mathrm{P}<0.05,{ }^{* *} \mathrm{P}<0.01,{ }^{* * *} \mathrm{P}<0.001$. Error bar, standard error. Bcl-2, B-cell lymphoma 2; Bak, Bcl-2 antagonist/killer 1; Bax, Bcl-2 associated X; Cx32, connexin 32; CCK-8, Cell Counting Kit-8; CDDP, cisplatin; siRNA, small interfering RNA.

The resistance of A2780/CDDP cells (RI=5.105) was identified by CCK-8 assay, western blot analysis and flow cytometry in the present study. Thus, the association between Cx32 expression and the three aforementioned factors involved in cisplatin resistance was investigated.
Alterations in the expression levels of drug transporters lead to cisplatin resistance by affecting the intracellular drug concentration $(21,22)$. Compared with the levels in A2780 cells, high expression levels of efflux transporters (MRP-2, ATP7A and ATP7B) and low expression of an influx transporter 
A

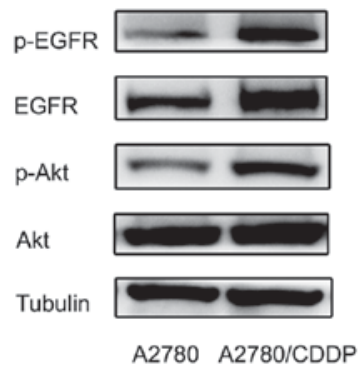

B

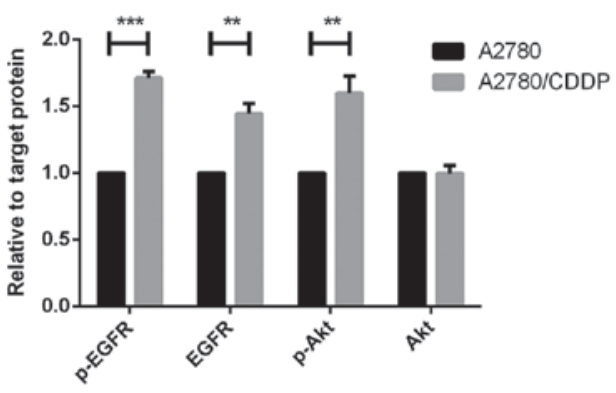

C
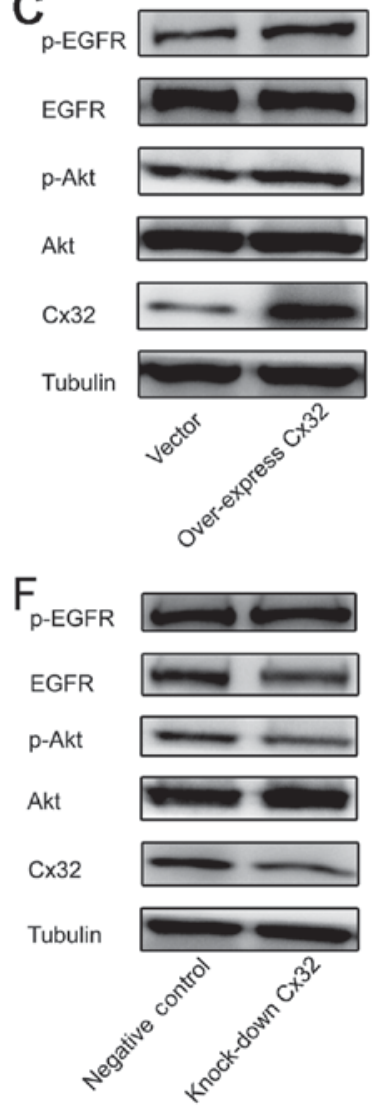

D

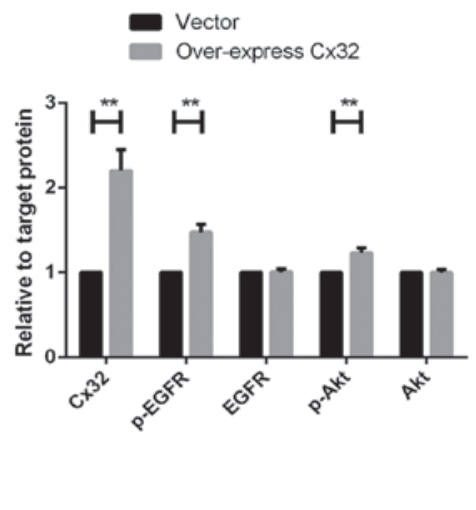

G

- Negative control Knock-down $\mathrm{C} \times 32$

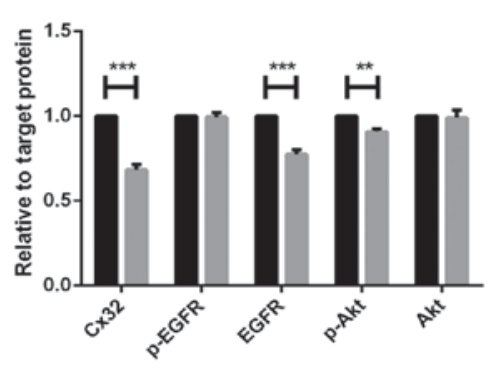

E

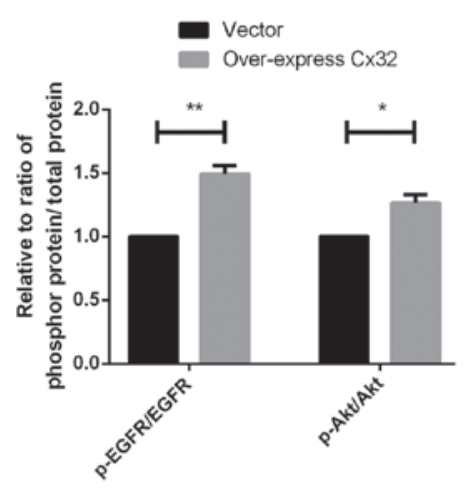

$\mathrm{H}$

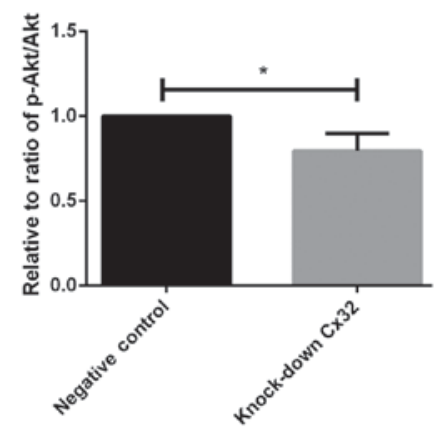

Figure 5. Cx32 promotes cisplatin resistance by activating the EGFR-Akt signalling pathway. (A and B) Expression of numerous proteins associated with the EGFR signalling pathway were compared between the A2780 cells and A2780/CDDP cells ( $n=3$ ). A2780/CDDP cells exhibited increased expression levels of p-EGFR, EGFR and p-Akt. (C-E) Following Cx32 overexpression in A2780 cells, numerous proteins associated with the EGFR-Akt signalling pathway were detected by western blotting (n=3). (F-H) Cx32 was knocked down by small interfering RNA-Cx32 in A2780/CDDP cells; several proteins associated with the EGFR-Akt signalling pathway were detected by western blotting $(\mathrm{n}=3) .{ }^{*} \mathrm{P}<0.05,{ }^{* *} \mathrm{P}<0.01,{ }^{* * * *} \mathrm{P}<0.001$. Error bar, standard error. Akt, protein kinase $\mathrm{B}$; Cx32, connexin 32; CDDP, cisplatin; EGFR, epidermal growth factor receptor; p, phosphorylated.

(CTR1) in A2780/CDDP cells were observed. Following the dysregulation of $\mathrm{Cx} 32$ expression, the expression levels of two cisplatin-associated efflux transporters (MRP-2 and ATP7A) were affected. $\mathrm{Cx}$-controlled expression of drug transporters has been reported in several studies. For example, Liu et al (23) reported that increasing $\mathrm{Cx} 43$ expression enhanced chemotherapy sensitivity in BGC-823 cells by downregulating the levels of P-glycoprotein; however, few studies have reported that $\mathrm{Cx} 32$ has such a function. The results of the present study suggested that Cx32-regulated the expression of drug efflux transporters, which may underlie cisplatin resistance.

Activation of the DNA repair system can counteract the fatal DNA damage caused by cisplatin (22). Compared with in A2780 cells, the expression of ERCC1 in A2780/CDDP cells was significantly higher in the present study. Regardless of whether
Cx32 was overexpressed in A2780 cells or downregulated in A2780/CDDP cells, the expression of ERCC1 was reduced. The expression levels of ERCC1, a key enzyme of nucleotide excision repair, participates in the DNA repair system $(24,25)$. Our previous study suggested that GJs reduced the function of the DNA repair system in cancer cells (26). Accordingly, it was proposed that overexpressing Cx32 in A2780 cells may enhance the function of GJs and weaken the activity of the DNA repair system, leading to reduced ERCC1 expression. Thus, the activation of the DNA repair system may not comprise the mechanism of Cx32-mediated cisplatin resistance.

Activation of anti-apoptotic pathways promotes the survival of cells following cisplatin treatment $(27,28)$. In the present study, the expression of cleaved caspase- 3 increased, and cisplatin cytotoxicity was enhanced following $\mathrm{Cx} 32$ 
knockdown. Conversely, Cx32 overexpression significantly increased cell chemoresistance to cisplatin and decreased the protein expression levels of cleaved caspase-3. The expression of Bcl-2 family members was also detected in the present study. Cx32 overexpression increased the expression of $\mathrm{Bcl}-2$ (an anti-apoptotic member) and decreased the expression of pro-apoptotic members (Bak and Bax), and vice versa. Based on these findings, Cx32 may participate in cisplatin resistance via its anti-apoptotic effect. In addition, the EGFR-Akt signalling pathway, known for its anti-apoptotic effect (29), exhibited variations in protein expression between A2780 and A2780/CDDP cells; expression was upregulated or downregulated via alterations in Cx32 expression. Therefore, it was hypothesized that $\mathrm{Cx} 32$ serves an anti-apoptotic role in cisplatin resistance by activating the EGFR-Akt signalling pathway.

Although numerous potential mechanisms of Cx32-mediated cisplatin resistance in human ovarian cancer were investigated, the most upstream mechanism of Cx32-mediated cisplatin resistance remains unclear. The present study was conducted using only one ovarian cancer cell line (A2780), which was of endometrioid ovarian cancer origin. Future studies on a variety of ovarian cancer cell lines, particularly the most clinically representative cell lines, may be of greater value. In addition, relevant clinical research data and xenograft animal model experiments are required for further investigation into the mechanisms underlying cisplatin resistance in ovarian cancer in the future.

Collectively, the findings of the present study indicated that $\mathrm{Cx} 32$ contributes to cisplatin resistance in human ovarian cancer cells via two mechanisms. Cx32 may be involved in cisplatin resistance by modulating the expression of drug efflux transporters and activating the EGFR-Akt anti-apoptosis axis. Thus, Cx32 may be a considered as a potential therapeutic target for overcoming cisplatin resistance in the treatment of human ovarian cancer.

\section{Acknowledgements}

Not applicable.

\section{Funding}

The present study was supported by the National Natural Science Foundation of China (grant no. 81473234), the Fundamental Research Funds for the Central Universities (grant no. 16ykjc01), the grant from Department of Science and Technology of Guangdong Province (grant no. 20160908), and the Joint Fund of the National Natural Science Foundation of China (grant no. U1303221).

\section{Availability of data and materials}

The datasets used and/or analysed during the current study are available from the corresponding author on reasonable request.

\section{Authors' contributions}

YZ performed western blotting, flow cytometry, plasmid transfection and siRNA interference experiments, and collected and analysed data, and wrote the manuscript. LT was involved in analysing the results and performing flow cytometry; LXF made substantial contributions in the acquisition and interpretation of the data. $\mathrm{KH}$ acquired the reagents and materials and was involved in performing western blotting and collecting data. HML performed the CCK- 8 assay and cell culture; HG contributed to the detection of protein expression; XW and QW made substantial contributions to the design of the present study. All authors read the paper, discussed the results and approved the final version.

\section{Ethics approval and consent to participate}

Not applicable.

\section{Patient consent for publication}

Not applicable.

\section{Competing interests}

The authors declare that they have no competing interests.

\section{References}

1. Cho KR and Shih IeM: Ovarian cancer. Annu Rev Pathol 4: 287-313, 2009.

2. Siegel RL, Miller KD and Jemal A: Cancer statistics, 2018. CA Cancer J Clin 68: 7-30, 2018.

3. Armstrong DK, Bundy B, Wenzel L, Huang HQ, Baergen R, Lele S, Copeland LJ, Walker JL and Burger RA; Gynecologic Oncology Group: Intraperitoneal cisplatin and paclitaxel in ovarian cancer. N Engl J Med 354: 34-43, 2006.

4. Ai Z, Lu Y, Qiu S and Fan Z: Overcoming cisplatin resistance of ovarian cancer cells by targeting HIF-1-regulated cancer metabolism. Cancer Lett 373: 36-44, 2016.

5. Agarwal R and Kaye SB: Ovarian cancer: Strategies for overcoming resistance to chemotherapy. Nat Rev Cancer 3: 502-516, 2003.

6. Wu W, Fan L, Bao Z, Zhang Y, Peng Y, Shao M, Xiang Y, Zhang X, Wang Q and Tao L: The cytoplasmic translocation of Cx32 mediates cisplatin resistance in ovarian cancer cells. Biochem Biophys Res Commun 487: 292-299, 2017.

7. Kanczuga-Koda L, Koda M, Sulkowski S, Wincewicz A, Zalewski B and Sulkowska M: Gradual loss of functional gap junction within progression of colorectal cancer-a shift from membranous CX32 and CX43 expression to cytoplasmic pattern during colorectal carcinogenesis. In Vivo 24: 101-107, 2010.

8. Kar R, Batra N, Riquelme MA and Jiang JX: Biological role of connexin intercellular channels and hemichannels. Arch Biochem Biophys 524: 2-15, 2012.

9. Kumar NM and Gilula NB: The gap junction communication channel. Cell 84: 381-388, 1996.

10. Yang J, Qin G, Luo M, Chen J, Zhang Q, Li L, Pan L and Qin S: Reciprocal positive regulation between Cx26 and PI3K/Akt pathway confers acquired gefitinib resistance in NSCLC cells via GJIC-independent induction of EMT. Cell Death Dis 6: e1829, 2015.

11. Yang Y, Zhu J, Zhang N, Zhao Y, Li WY, Zhao FY, Ou YR, Qin SK and Wu Q: Impaired gap junctions in human hepatocellular carcinoma limit intrinsic oxaliplatin chemosensitivity: A key role of connexin 26. Int J Oncol 48: 703-713, 2016.

12. Murphy SF, Varghese RT, Lamouille S, Guo S, Pridham KJ, Kanabur P, Osimani AM, Sharma S, Jourdan J, Rodgers CM, et al: Connexin 43 inhibition sensitizes chemoresistant glioblastoma Cells to Temozolomide. Cancer Res 76: 139-149, 2016.

13. Wang SQ, Zhang SW, Zhang CZ, Zhao ZY and Wang YJ: Connexin 43 enhances oxaliplatin cytotoxicity in colorectal cancer cell lines. Cell Mol Biol 63: 53-58, 2017.

14. Zhao Y, Lai Y, Ge H, Guo Y, Feng X, Song J, Wang Q, Fan L, Peng Y, Cao M, et al: Non-junctional Cx32 mediates anti-apoptotic and pro-tumor effects via epidermal growth factor receptor in human cervical cancer cells. Cell Death Dis 8: e2773, 2017. 
15. Lai Y, Fan L, Zhao Y, Ge H, Feng X, Wang Q, Zhang X, Peng Y, Wang $X$ and Tao L: Cx32 suppresses extrinsic apoptosis in human cervical cancer cells via the NF- $\mathrm{BB}$ signalling pathway. Int J Oncol 51: 1159-1168, 2017.

16. Wu Q, Yang Z, Nie Y, Shi Y and Fan D: Multi-drug resistance in cancer chemotherapeutics: Mechanisms and lab approaches. Cancer Lett 347: 159-166, 2014.

17. Kathawala RJ, Gupta P, Ashby CR Jr and Chen ZS: The modulation of $\mathrm{ABC}$ transporter-mediated multidrug resistance in cancer: A review of the past decade. Drug Resist Updat 18: 1-17, 2015.

18. O'Grady S, Finn SP, Cuffe S, Richard DJ, O'Byrne KJ and Barr MP: The role of DNA repair pathways in cisplatin resistant lung cancer. Cancer Treat Rev 40: 1161-1170, 2014.

19. Kim IS, Ganesan P and Choi DK: Cx43 mediates resistance against $\mathrm{MPP}^{+}$-induced apoptosis in SH-SY5Y neuroblastoma cells via modulating the mitochondrial apoptosis pathway. Int J Mol Sci 17: pii: E1819, 2016.

20. Galluzzi L, Senovilla L, Vitale I, Michels J, Martins I, Kepp O, Castedo M and Kroemer G: Molecular mechanisms of cisplatin resistance. Oncogene 31: 1869-1883, 2012.

21. Amable L: Cisplatin resistance and opportunities for precision medicine. Pharmacol Res 106: 27-36, 2016.

22. Holohan C, Van Schaeybroeck S, Longley DB and Johnston PG: Cancer drug resistance: An evolving paradigm. Nat Rev Cancer 13: 714-726, 2013.
23. Liu D, Zhou H, Wu J, Liu W, Li Y, Shi G, Yue X, Sun X, Zhao Y, $\mathrm{Hu} \mathrm{X}$, et al: Infection by $\mathrm{Cx} 43$ adenovirus increased chemotherapy sensitivity in human gastric cancer BGC-823 cells: Not involving in induction of cell apoptosis. Gene 574: 217-224, 2015.

24. Takahata C, Masuda Y, Takedachi A, Tanaka K, Iwai S and Kuraoka I: Repair synthesis step involving ERCC1-XPF participates in DNA repair of the Top1-DNA damage complex. Carcinogenesis 36: 841-851, 2015.

25. McNeil EM and Melton DW: DNA repair endonuclease ERCC1-XPF as a novel therapeutic target to overcome chemoresistance in cancer therapy. Nucleic Acids Res 40: 9990-10004, 2012.

26. Zhang Y, Tao L, Fan L, Peng Y, Yang K, Zhao Y, Song Q and Wang Q: Different gap junction-propagated effects on cisplatin transfer result in opposite responses to cisplatin in normal cells versus tumor cells. Sci Rep 5: 2563, 2015.

27. Norouzi-Barough L, Sarookhani MR, Sharifi M,Moghbelinejad S, Jangjoo S and Salehi R: Molecular mechanisms of drug resistance in ovarian cancer. J Cell Physiol 233: 4546-4562, 2018.

28. Mohammad RM, Muqbil I, Lowe L, Yedjou C, Hsu HY, Lin LT, Siegelin MD, Fimognari C, Kumar NB, Dou QP, et al: Broad targeting of resistance to apoptosis in cancer. Semin Cancer Biol 35 (Suppl): S78-S103, 2015.

29. Seshacharyulu P, Ponnusamy MP, Haridas D, Jain M, Ganti AK and Batra SK: Targeting the EGFR signaling pathway in cancer therapy. Expert Opin Ther Targets 16: 15-31, 2012. 